\title{
Effect of duty cycles of tumor-treating fields on glioblastoma cells and normal brain organoids
}

\author{
EUNBI YE $^{1 *}$, JUNG EUN LEE $^{2 *}$, YOUNG-SOO LIM $^{1}$, SEUNG HO YANG $^{2}$ and SUNG-MIN PARK ${ }^{1,3,4}$ \\ ${ }^{1}$ Department of Convergence IT Engineering, Pohang University of Science and Technology, Pohang-si, \\ Gyeongsangbuk-do 37673; ${ }^{2}$ Department of Neurosurgery, St. Vincent's Hospital, College of Medicine, The Catholic University \\ of Korea, Suwon-si, Gyeonggi-do 16247; Departments of ${ }^{3}$ Electrical Engineering and ${ }^{4}$ Mechanical Engineering, \\ Pohang University of Science and Technology, Pohang-si, Gyeongsangbuk-do 37673, Republic of Korea
}

Received August 23, 2021; Accepted December 9, 2021

DOI: $10.3892 /$ ijo.2021.5298

\begin{abstract}
Tumor-treating fields (TTFields) are emerging cancer therapies based on alternating low-intensity electric fields that interfere with dividing cells and induce cancer cell apoptosis. However, to date, there is limited knowledge of their effects on normal cells, as well as the effects of different duty cycles on outcomes. The present study evaluated the effects of TTFields with different duty cycles on glioma spheroid cells and normal brain organoids. A customized TTFields system was developed to perform in vitro experiments with varying duty cycles. Three duty cycles were applied to three types of glioma spheroid cells and brain organoids. The efficacy and safety of the TTFields were evaluated by analyzing the cell cycle of glioma cells, and markers of neural stem cells (NSCs) and astrocytes in brain organoids. The application of the TTFields at the 75 and $100 \%$ duty cycle markedly inhibited the proliferation of the U87 and U373 compared with the control. FACS analysis revealed that the higher the duty cycle of the
\end{abstract}

Correspondence to: Professor Seung Ho Yang, Department of Neurosurgery, St. Vincent's Hospital, College of Medicine, The Catholic University of Korea, 93 Jungbudaero, Paldal-gu, Suwon-si, Gyeonggi-do 16247, Republic of Korea

E-mail: 72ysh@catholic.ac.kr

Professor Sung-Min Park, Department of Convergence IT Engineering, Pohang University of Science and Technology, 77 Cheongam-ro, Nam-gu, Pohang-si, Gyeongsangbuk-do 37673, Republic of Korea

E-mail: sungminpark@postech.ac.kr

*Contributed equally

Abbreviations: 2D,two-dimensional; 3D, three-dimensional; bFGF, basic fibroblast growth factor; EB, embryoid body; EGF, epidermal growth factor; GB, glioblastoma; iPSCs, induced pluripotent stem cells; NSC, neural stem cell; PDMS, polydimethylsiloxane; ROCK, Rho-associated kinase; TTFields, tumor-treating fields

Key words: 3D cell culture, TTFields, electromagnetic field, computational simulation, neurotoxicity applied fields, the greater the increase in apoptosis detected. Exposure to a higher duty cycle resulted in a greater decrease in NSC markers and a greater increase in glial fibrillary acidic protein expression in normal brain organoids. These results suggest that TTFields at the 75 and $100 \%$ duty cycle induced cancer cell death, and that the neurotoxicity of the TTFields at $75 \%$ was less prominent than that at $100 \%$. Although clinical studies with endpoints related to safety and efficacy need to be performed before this strategy may be adopted clinically, the findings of the present study provide meaningful evidence for the further advancement of TTFields in the treatment of various types of cancer.

\section{Introduction}

Tumor-treating fields (TTFields) is an emerging cancer treatment modality that uses alternating, low intensity $(1-3 \mathrm{~V} / \mathrm{cm})$ electric fields at intermediate frequencies $(100-300 \mathrm{KHz})$ to disrupt cancer cell proliferation (1-3). In initial in vitro experiments conducted in the early 2000s, the application of TTFields to various types of tumor cell lines was found to exert inhibitory effects on growth and induced cell cycle arrest and apoptosis (1). The anticancer efficacy of TTFields was further demonstrated by clinical studies, and the TTFields system rapidly received FDA approval for newly diagnosed and recurrent glioblastoma (GB) $(4,5)$. Clinical studies have demonstrated that the use of TTFields in combination with chemotherapy improves progression-free, overall and long-term survival compared with chemotherapy alone (4-6). The combination of TTFields and immunotherapy may exert a synergistic effect as cellular stress signals induced by TTFields facilitate immune activation and immunogen-induced cell death (7). However, mechanistic investigations are required to optimize the use of TTFields in combination with additional modalities, including radiation therapy.

Although the underlying mechanisms are under debate, TTFields reportedly target mitosis and cytokinesis (8-10). By inhibiting mitosis and cytokinesis, TTFields rapidly target proliferating cells only, which results in tumor specificity. Therefore, they are considered to cause more significant damage to cancer cells than normal cells. TTFields align proteins possessing large dipole moments essential for cell 
division, such as tubulin dimers (8) and septins (9), which interferes with spindle alignment. Consequently, TTFields induce mitotic catastrophe, leading to cell cycle arrest at the $\mathrm{G} 2 / \mathrm{M}$ phase and culminating in cell death $(1,10)$. In addition, cells dividing parallel to the externally applied field are more affected by the field than cells dividing in other directions (11). Other biological mechanisms considered to be involved in the effects of TTFields include apoptosis, autophagy, DNA repair and immunogenic cell death $(11,12)$. Moreover, computational studies have reported that the dielectrophoretic forces are not sufficient to exert a significant effect on tubulin and septins, while possibly affecting cellular molecules $(13,14)$. This contradiction suggests that there remains a need for in-depth biological studies to elucidate the mechanisms of action of TTFields.

Although monolayer cell cultures are frequently used in mechanism studies for TTFields, two-dimensional (2D) cell cultures are oversimplified versions of tumors and do not recapitulate in vivo cellular organization and interactions $(15,16)$. The microenvironment of tumor spheroids resembles that of tumors more closely and may thus be a more suitable in vitro cancer model than monolayer cultures. These properties of tumor spheroids confer anticancer drug resistance and radiation resistance to tumor spheroids, as observed in human cancers. The present study, to the best of our knowledge, is the first to apply TTFields to three-dimensional (3D) glioma spheroids.

The use of 3D cell models in 3D culture environments based on induced pluripotent stem cells (iPSCs) has enabled the study of organs in vitro. Brain organoid formation relies on the self-organization ability of iPSCs, which develop into organized structures that resemble distinct regions of the brain, maintaining hallmarks of key developmental processes involved in brain formation $(17,18)$. Thus, 3D brain organoids may be a realistic 3D model which can be used to minimize the gap between 2D cell cultures and animal models. The present study used brain organoids as a novel platform to evaluate the effects of TTFields on normal brain cells.

As the patient is required to wear the TTFields device for long periods of time, the main design concern for improving usability for the patient is that it can be worn more comfortably. Increased compliance with TTFields therapy has been reported to be an independent prognostic factor for improved survival in GB $(19,20)$. In general, the longer the duration of the application of TTFields, the more prominent the therapeutic effect; therefore, patients are advised to wear the device for impractically long periods of time ( $23 \mathrm{~h}$ /day with $100 \%$ duty cycle), without any parametric evidence. A previous clinical study revealed the longer survival of patients with recurrent GB multiforme (GBM) treated with TTFields for $18 \mathrm{~h} /$ day compared with those treated for $<18 \mathrm{~h} /$ day (19). In addition, patients with a compliance of $>20 \mathrm{~h} /$ day have been shown to exhibit extended survival rates (20). As the TTFields device requires a high compliance rate, it is designed to be wearable and portable, with minimal impact on daily activities. Thus, a further improvement in the usability of the system, such as device miniaturization and operation time extension, is imperative to benefit a greater number of patients. The battery determines the size and weight of the device. Therefore, a method for efficient power management for the TTFields system is required (21).
The present study noted that energy efficiency can be further improved through the regulation of the duty cycle. The duty cycle is the ratio of time a load or circuit is 'on' compared to the time the load or circuit is 'off' per minute, which is the typical definition for the duty cycle of any electronic device. Previous studies $(19,20)$ have investigated the number of hours per day of wearing the device, but not the duty cycle that was explored herein. TTFields with tumor-treating effects are advantageous even if the duty cycle is lowered. First, energy consumption can be reduced by adjusting the duty cycle, which in turn is associated with a smaller and lighter battery, and prolongs the device operation time without requiring recharging. Furthermore, it will help advance the TTFields device into more suitable forms, such as rechargeable implantable systems. These forms would maximize the usability as patients wish to disguise the worn device. Currently, patients often use wigs and hats; however, the cables connecting the power supply and field generator to the transducer array are intrusive and noticeable (22). However, there is a lack of agreement on the duty cycle, which may be the key for this form factor evolution. Therefore, the present study attempted to establish an association between the duty cycle and treatment effect of TTFields as the first step towards the further enhancement of the TTFields system.

The present study aimed to evaluate the treatment efficacy and safety of TTFields using three glioma cell lines and normal brain organoids. In addition, the present study evaluated whether the duty cycles of TTFields affected their therapeutic efficacy. To the best of our knowledge, there is no study available to date on the duty cycle of TTFields. Herein, experiments were conducted on three duty cycles and a control group, and all experiments were conducted for $72 \mathrm{~h}$, which is the typical duration for TTFields experiments (23). For the in vitro experiment, a system was designed that generates an electric field suitable for TTFields in a cell culture environment based on numerical electromagnetic simulations and a simple TTFields system was fabricated that can modulate the duty cycle freely. In the present study, details of TTF-induced cell damage to tumor spheroid cells and normal brain organoids were analyzed and compared according to the duty cycle.

\section{Materials and methods}

TTFields system design. The customized stimulation system is illustrated in Fig. 1A. This system can be easily constructed in a laboratory environment as all components are readily available. Commands were delivered to the microcontroller included in a custom-built circuit to adjust the TTFields parameters and the temperature was monitored using a computer. The custom-built circuit generated a $200 \mathrm{KHz}$ sine wave and a 2.5 Peak-to-peak voltage (Vpp) amplitude was fixed to the electrode plate. A system stability test of 7 days (well beyond the in vitro testing period) revealed no interference errors in the circuit and output signal. During the experimental period, the cell plate was kept in an incubator at $37^{\circ} \mathrm{C}$ with $5 \% \mathrm{CO}_{2}$. The gap between the cell culture plate and the lid was sealed with Bemis parafilm to prevent the evaporation of the cell medium. The wire connected to the electrode came out through a small gap in the incubator door. The detailed configuration of the custom-built circuit is illustrated in 


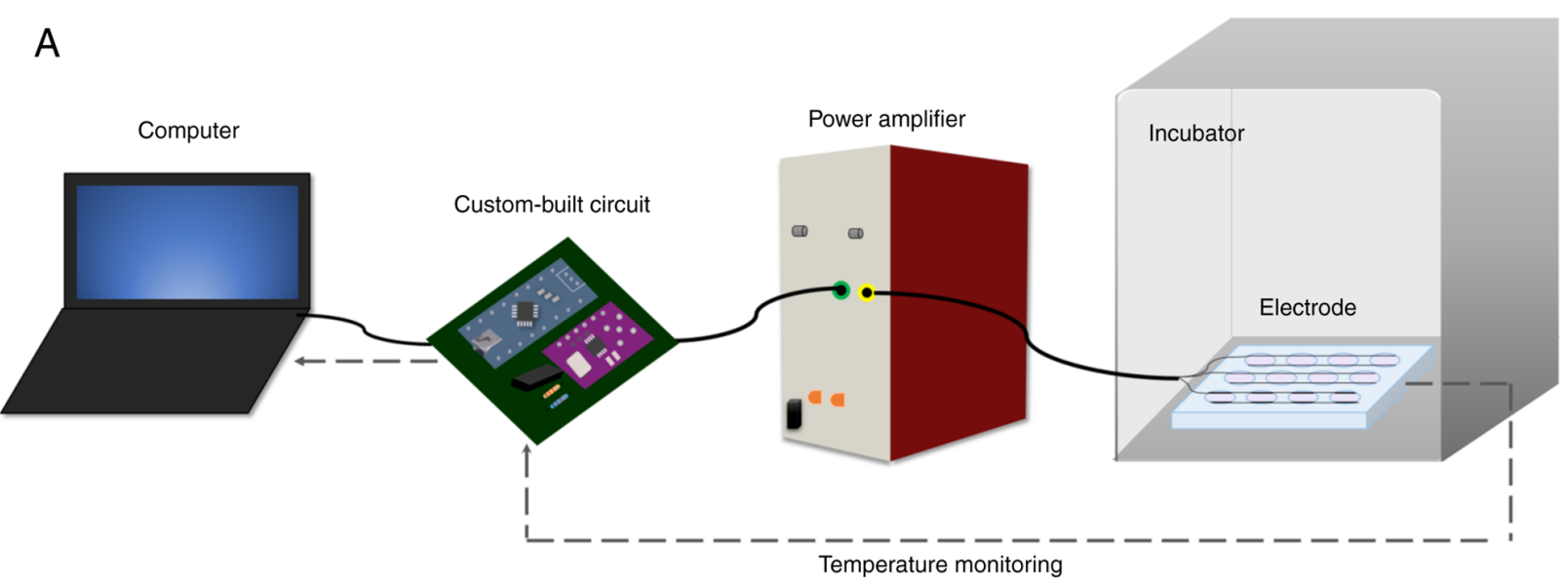

B

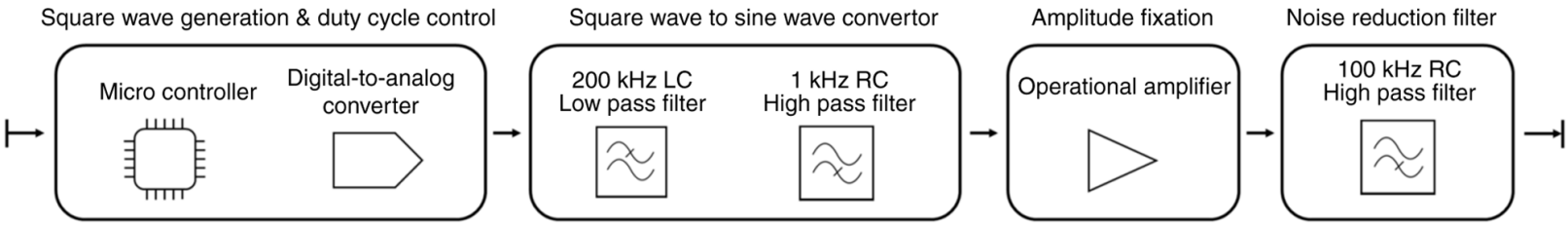

C

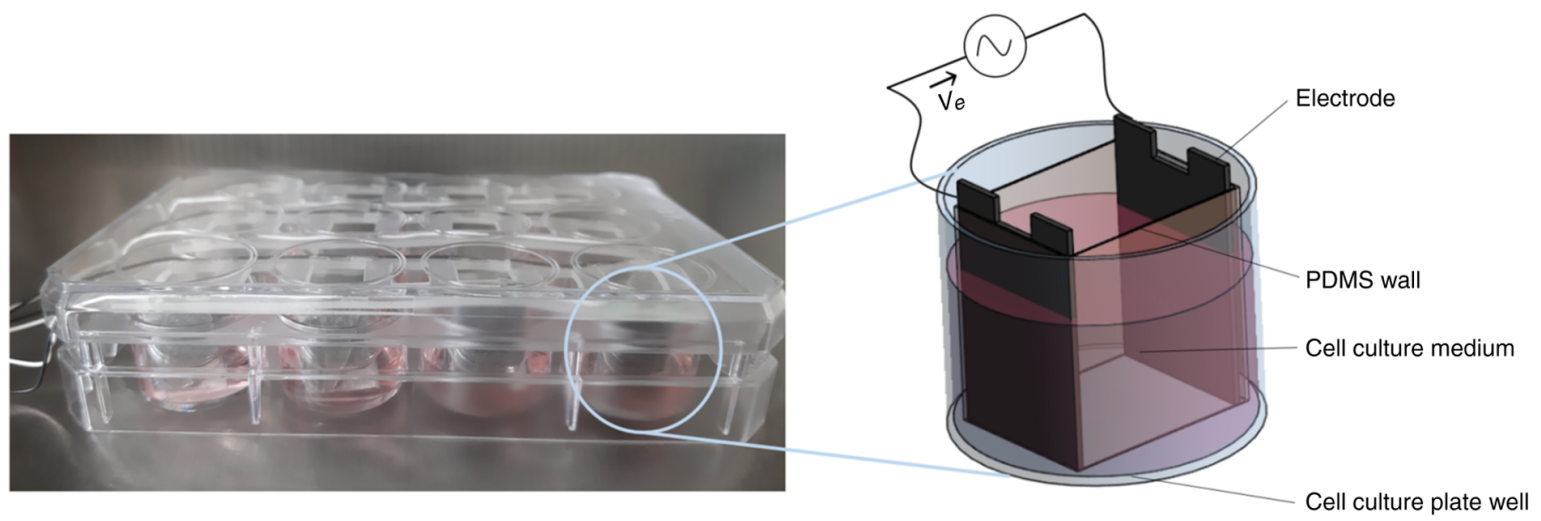

Figure 1. Tumor-treating fields application. (A) Schematic diagram of the experimental setup. (B) Custom-built $200 \mathrm{KHz}$ sine wave generator circuit. (C) Electrode design ( $\mathrm{Ve}=2.5 \mathrm{Vpp})$ and detailed view.

Fig. 1B. The control software was developed using Arduino IDE software version 1.8.13 (Arduino). Square-wave generation and duty cycle control were performed using a microcontroller unit (Arduino Nano V3.0; Arduino). A digital-to-analog control microprocessor (CJMCU-9833; Shenzhen Bixinda Technology Co., Ltd.) was used to create a $200 \mathrm{KHz}$ square wave and control the duty from the microcontroller unit signal. Square wave to sine wave conversion was performed using our custom built $1 \mathrm{KHz}$ RC high-pass filter and $200 \mathrm{KHz}$ LC low-pass filter. The RC high-pass filter removes the direct current offset; the LC low-pass filter converts a square wave to a sine wave. For amplitude control and buffer, a high-frequency response was required. Amplitude fixation was performed using an operational amplifier (MC34074; ON Semiconductor Corp.) as the amplifier and buffer. The noise reduction filter used a $100 \mathrm{KHz}$ RC high-pass filter and was placed at the end of the generator immediately before the power amp input. The custom-built $200 \mathrm{KHz}$ sine wave generator circuit is portable, measuring 105x150x55 mm, and weighs $260 \mathrm{~g}$.

The TTFields electrodes were fabricated using a 12-mm-wide, 19-mm-long and 0.5-mm-thick stainless-steel plate, and the distance between the pair of electrodes per well was $15 \mathrm{~mm}$. The upper part of the electrode was fixed to a holder made using a 3D printer (Fig. 1C), and four pairs of electrodes, corresponding to one row of a 12-well plate, were connected in parallel. Each pair of electrodes were wrapped using polydimethylsiloxane (PDMS) to prevent spheroid cells from leaking out from the uniform field zone.

Heating should be minimized to avoid the confounding effects of temperature rise and TTFields on cancer cells. To evaluate the level of heating induced by TTFields on the cell culture medium, temperature was recorded in the culture medium during the application of TTFields at the $100 \%$ duty cycle as the 'worst-case scenario'. A digital thermometer (DS18B20; Maxim Integrated) was used to monitor the temperature of the well once every minute. Little to no change in temperature was induced by TTFields ( $\mathrm{Ve}=2.5 \mathrm{Vpp}$ ) for $72 \mathrm{~h}$, indicating that the confounding effect of heating on the cell culture medium is unlikely to be observed during the in vitro testing.

Computational simulation. Numerical computations of electric field distribution and the resulting temperature increment 
in the cell culture medium were performed using Sim4Life ver. 6.0 (ZMT Zurich MedTech AG). To identify the electric field, a quasi-static electromagnetic solver (included with the aforementioned software) was used to calculate low-frequency electromagnetic problems (24) using the following equation (Equation 1):

$$
\nabla \cdot \tilde{\epsilon} \nabla \varnothing=0
$$

where ' $\varepsilon$ ' is the complex electric permittivity and ' $\phi$ ' is a scalar potential. The electromagnetic field was recorded for use as a heat source for thermal simulations.

The temperature change in the tissues during thermal ablation was calculated using the Pennes bioheat equation (Equation 2), which has been used to solve computational

$$
\begin{gathered}
\rho c \frac{\partial T}{\partial t}=\nabla \cdot(k \nabla T)+\rho Q+\rho S-\rho_{b} \rho \omega\left(T-T_{b}\right) \\
\rho c \frac{\partial T}{\partial t}=\nabla \cdot(k \nabla T)+\rho Q+\rho S
\end{gathered}
$$

bioelectromagnetic problems since its formulation (25): where ' $\rho$ ' is material density, ' $c$ ' is specific heat capacity, ' $T$ ' is tissue temperature, ' $\mathrm{k}$ ' is thermal conductivity, ' $\mathrm{Q}$ ' is metabolic heat generation rate, ' $S$ ' is the specific absorption rate and ' $\omega$ ' is the perfusion rate. The term ' $\rho_{b} c_{b} \rho w$ ' is sometimes referred to as the heat transfer rate by blood perfusion. As the present study did not need to consider the effect of blood in Equation (2), this was thus simplified to Equation (3).

For simulation, the conductivities of PDMS, stainless steel and cell culture plate wall were assigned values of $2.5 \times 10^{-14}$, $1.1 \times 10^{6}$ and $5 \times 10^{-4} \mathrm{~S} / \mathrm{m}$, respectively. The conductivity of the cell culture medium was determined at $1.8 \mathrm{~S} / \mathrm{m}$ using a conductivity meter (CP-50N; Isteck, Inc.) and the conductivity of the spheroid tumor cell was specified as $0.24 \mathrm{~S} / \mathrm{m}$ (26). For properties other than conductivity, the values provided by the software were used.

Cell preparation: Tumor spheroid cell culture. U87 (glioblastoma of unknown origin) were purchased from ATCC (Lot. no. 60173414) and U373 MG ATCC were purchased from the Korean Cell line Bank (Lot no. 22741). All cell lines had been authenticated using short tandem repeat profiling. They were transduced with a lentiviral construct containing the Firefly luciferase gene as previously described (27). The U251 cell line was purchased was originally obtained from Sigma-Aldrich; Merck KGaA. The cells were cultured in suspension at 100 cells/well in 6-well plates for 14 days in a neural stem cell (NSC) medium, consisting of Neurobasal (Thermo Fisher Scientific, Inc.) and DMEM/F12 media (HyClone; Cytiva) (1:1) supplemented with 1 x B27, $1 \times \mathrm{N} 2$, basic fibroblast growth factor (bFGF, $20 \mathrm{ng} / \mathrm{ml}$ ), and epidermal growth factor (EGF, $20 \mathrm{ng} / \mathrm{ml}$ ). The number of neurospheres per well was determined by counting the neurospheres in five wells on days 7 and 14 . The cell density was adjusted to $1 \times 10^{5}$ cells $/ \mathrm{ml}$ cell culture medium using a cell counter and $200 \mu \mathrm{l}$ of the cell culture was plated into the wells of the sterile 96-well cell culture plate. Neurospheres with diameters $>50 \mu \mathrm{m}$ were counted. Bioluminescence was detected using an in vivo imaging system (IVIS 200; Xenogen Corporation).
Generation of human brain organoids. CMC-hiPSC-011 cells (28) were kindly provided by the laboratory of Dr Joo (the Catholic University of Korea, https://nih.go.kr/contents. es?mid=a50401110300). Organoids were generated using the STEMdiff Cerebral Organoid kit (08570; STEMCELL Technologies) following the manufacturer's instructions. On day 0, CMC-hiPSC-011 cells at 90\% confluency were dissociated into single cells using Accutase (A1110501, Gibco; Thermo Fisher Scientific, Inc.) for $5 \mathrm{~min}$ at $37^{\circ} \mathrm{C}$. Following centrifugation at $1,000 \mathrm{x}$ g for $5 \mathrm{~min}$ at room temperature, the iPSCs were resuspended in embryoid body (EB) formation medium (05893, STEMCELL Technologies) with $10 \mu \mathrm{M}$ Y27632 (Y503; Sigma-Aldrich; Merck KGaA), a Rho-associated kinase (ROCK) inhibitor, Y-27632, and diluted to a concentration of $9 \times 10^{3}$ cells $/ \mathrm{ml}$. Subsequently, $100 \mu \mathrm{l}$ cell suspension were distributed into each well of a low-attachment 96-well U-bottom plate (Corning, Inc.) to form single EBs, and the medium was changed every 2 days. On days 5-6, one-half of the medium was replaced with an induction medium. On day 7, organoids were harvested, embedded in Matrigel (Corning, Inc.), and grown in expansion medium in suspension culture in ultra-low attachment 6-well plates (Corning, Inc.). The embedded organoids were maintained for 3 days and cultured in maturation medium, and the plates were transferred to a shaker for continuous culturing. The medium was changed every 3 days.

TTFields treatment and evaluation: TTFields application. The present study developed an in-house TTFields system that allows for the accurate adjustment of the duty cycle, while maintaining a constant voltage of $2.5 \mathrm{Vpp}$ between electrode plates. TTFields of three duty cycles were applied to the cells. At this time, the duty cycle was adjusted based on 1 min (e.g., $30 \mathrm{sec}$ 'on' and $30 \mathrm{sec}$ 'off' in the case of 50\% duty). The cells were placed within the PDMS wall and exposed to the electric field generated between the electrodes. Three to five glioma spheroids were placed in each well. Three brain organoids matured for 40 days were placed per well. TTFields were applied for 3 days without changing the culture medium.

Cell cycle analysis. Following $72 \mathrm{~h}$ of TTFields treatment, floating cells from the medium were harvested by centrifugation at $230 \mathrm{x} \mathrm{g}$ for $30 \mathrm{sec}$ at room temperature, and adherent cells were dissolved through trypsinization (R001100, Gibco; Thermo Fisher Scientific, Inc.). Both cell populations were washed once with $5 \mathrm{ml}$ ice cold PBS, combined and resuspended in $500 \mu \mathrm{l}$ PBS. For cell cycle analysis, the cells were fixed in $4 \mathrm{ml}$ ice-cold 70\% ethanol (J.T. Baker) and the cell pellet was re-suspended in $150 \mu 1$ propidium iodide (Sigma-Aldrich; Merck KGaA) staining solution. The stained sample was incubated in a $37^{\circ} \mathrm{C}$ incubator for 40 min. The DNA content was analyzed using a flow cytometer (BD FACS Canto 2.0, BD Biosciences) and evaluated using Flowing Software 2.5.1 (University of Turku, Turku, Finland).

$H \& E$ staining. The sections were fixed with $4 \%$ paraformaldehyde in $0.1 \mathrm{M}$ phosphoric acid buffer (PB, pH 7.4). Samples containing paraffin were cut into tubular $8-\mu \mathrm{m}$-thick sections using a microtome and mounted on the APS coating slide. 
A

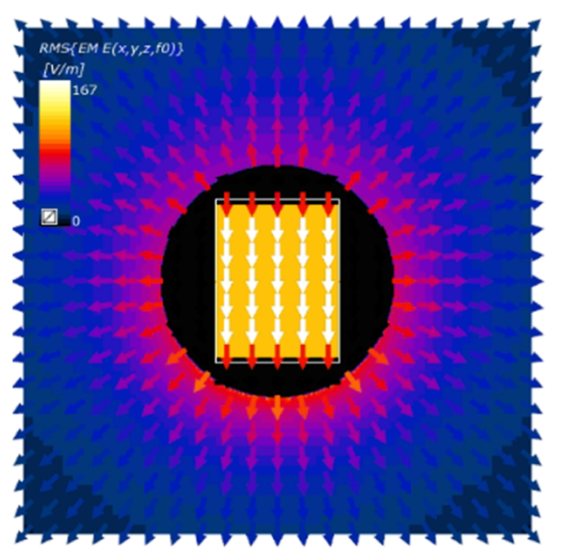

$\mathrm{B}$

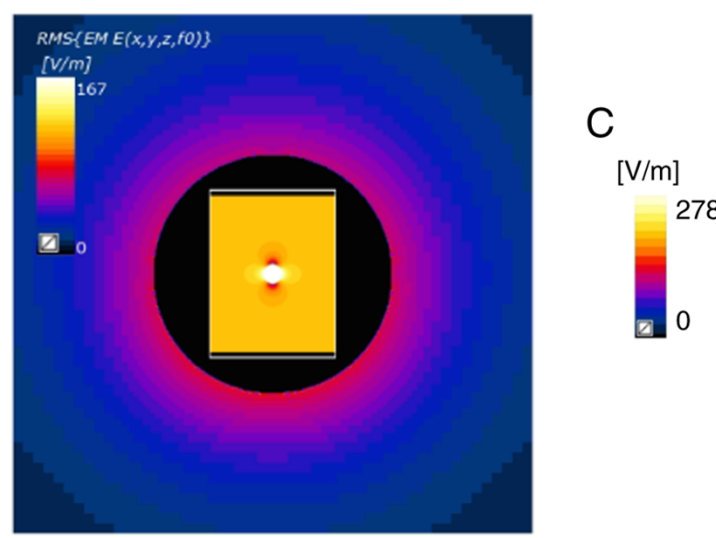

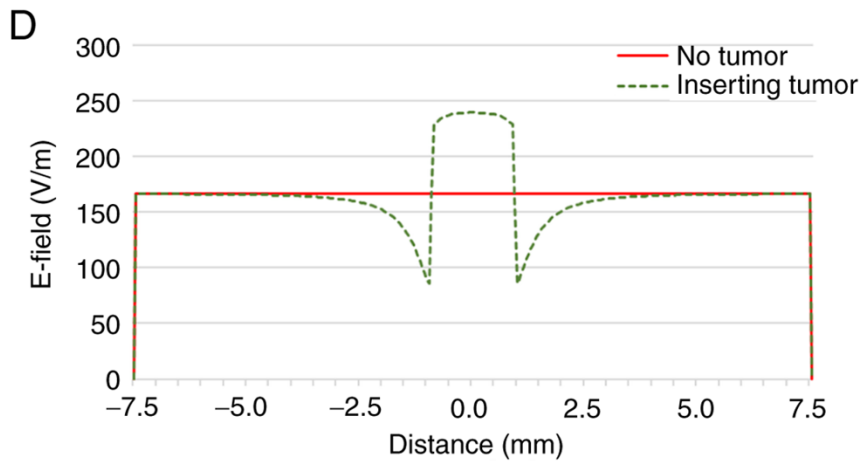

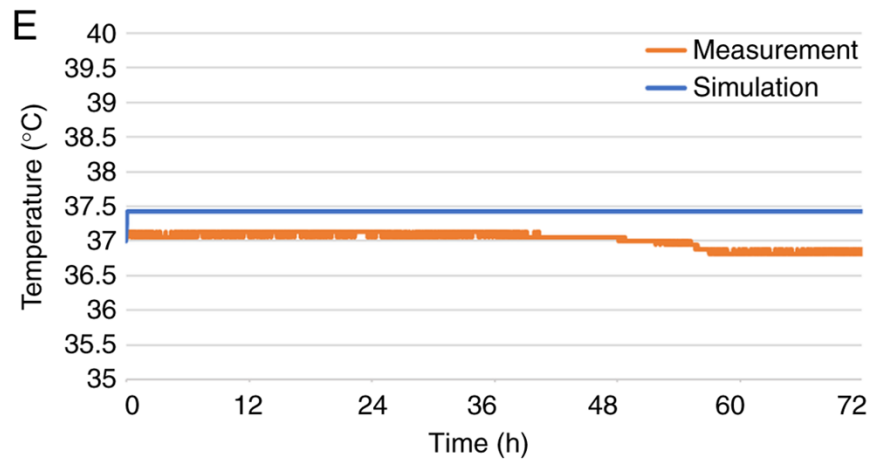

Figure 2. Computed electric field distribution and measured temperature rise within the representative well. (A) Computationally modeled XY plane of electric field strength (V/m; color bar) and vector (arrows). (B) Computationally modeled XY plane of electric field strength (V/m; colors) with the inserted tumor spheroid cell ( $\mathrm{r}=1 \mathrm{~mm})$. (C) Surface view of electric field distribution of the tumor spheroid cell. (D) Electric field distribution between electrodes (d=15 mm) with or without the tumor cell. (E) Temperature change calculated by simulation and measured during the experiment.

The sections were then deparaffinized and stained with hematoxylin and eosin (MilliporeSigma). The stained areas were dried using an ethanol series, washed with xylene and covered. Images were obtained using an upright optical microscope equipped with a digital camera (DP70, Olympus Corporation).

Immunofluorescence staining. The human brain organoids were fixed with $4 \%(\mathrm{w} / \mathrm{v})$ PFA, OCT-embedded, and cut into $8-\mu \mathrm{m}$-thick sections using a freezing microtome (Leica Microsystems $\mathrm{GmbH}$ ). The sections were blocked with $1 \%(\mathrm{w} / \mathrm{v})$ normal goat serum (Jackson ImmunoResearch Laboratories, Inc.) and incubated first at room temperature for $2 \mathrm{~h}$ with primary anti- $\beta$-III tubulin (1:500, 801201, BioLegend, Inc.), anti-Nestin (1:500, sc-23927, Santa Cruz Biotechnology Inc.), anti-glial fibrillary acidic protein (GFAP; 1:500, AB5804, Merck Millipore), anti-NeuN (1:200, ABN78, Merck Millipore) and anti-SOX2 (1:500, 3579, Cell Signaling, Inc.) antibodies, and subsequently with goat anti-mouse or rabbit Alexa Fluor 488 or 546 antibodies (1:1,000; Molecular Probes; Thermo Fisher Scientific, Inc.). Nuclei were labeled with DAPI (1:1,000, Sigma-Aldrich; Merck KGaA) at room temperature for $10 \mathrm{~min}$, and fluorescence was observed using a Zeiss LSM510 confocal microscope (Zeiss AG).

Statistical analysis. All data are expressed as the mean \pm SD or mean \pm SEM. Data were analyzed using an unpaired Student's t-test when comparing two groups. ANOVA with a post hoc Tukey's multiple comparisons test was used to determine whether differences between groups were statistically significant. A value of $\mathrm{P}<0.05$ was considered to indicate a statistically significant difference.

\section{Results}

Electromagnetic and thermal simulation. The computed electric field distribution and measured temperature rise in a well is illustrated in Fig. 2. These results confirm that the current system generates a homogeneous electric field between the electrodes, and the induced temperature increase resulting from this electric field is negligible (Fig. 2). The designed electrode generated a homogeneous electric field of $167 \mathrm{~V} / \mathrm{m}$ between the electrodes (Fig. 2A and D). When tumor cells were inserted, the electric field was concentrated in the tumor cells, increasing up to $272 \mathrm{~V} / \mathrm{m}$ (Fig. $2 \mathrm{~B}$ and D). The electric field formed on the surface of the tumor cell was examined (Fig. 2C). The temperature increment was verified using two methods: Through simulation and experimental measurements (Fig. 2E). While the temperature calculated for the cell culture medium revealed saturation after increasing by $0.4^{\circ} \mathrm{C}$, the measured temperature only fluctuated within the accuracy of the thermal sensor (DS18B20; Maxim Integrated) of $\pm 0.5^{\circ} \mathrm{C}$ (29). These results confirm that the system used herein does not induce heating during the in vitro experiments.

TTFields application on cancer cells. The bioluminescence intensity of glioma spheroids (U87-Luc and U373-Luc) was 

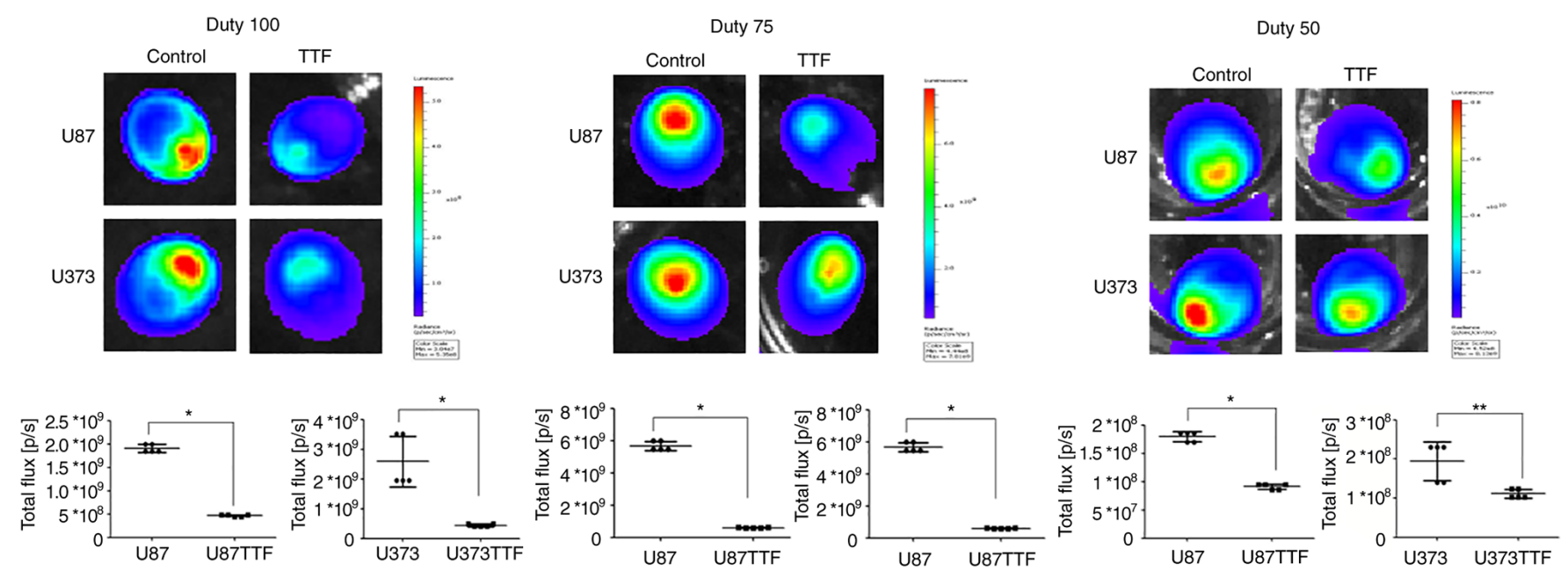

Figure 3. Bioluminescence imaging of U87 and U373 cells after applying tumor-treating fields for $72 \mathrm{~h}$. Data are presented as the average \pm SD (n=5). ${ }^{*} \mathrm{P}<0.001$ and ${ }^{* *} \mathrm{P}<0.05$. TTF, treated with tumor-treating fields.
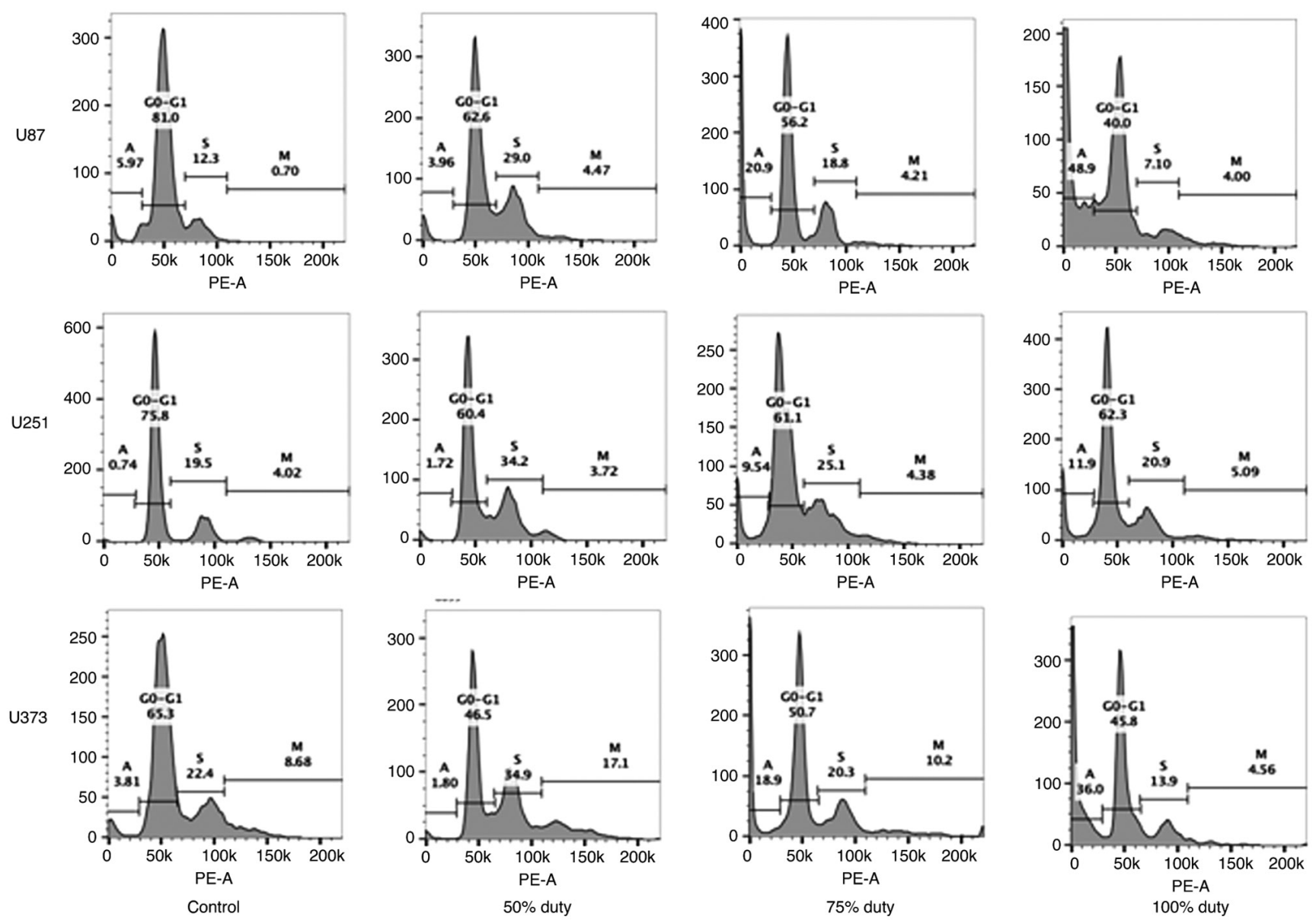

Figure 4. FACS analysis measured percentage of cells in the sub-G1 (dead cells), G0-G1, S, and M phases.

evaluated after applying TTFields. The bioluminescence intensity in the U87 and U373 cells treated with the TTFields was significantly lower than that in the control (Fig. 3 and Table SI). At $100 \%$ duty, the mean intensities in the U87 and $\mathrm{U} 373$ cells were $0.469 \mathrm{E}+09$ and $0.442 \mathrm{E}+09$ compared with the control at $1.90 \mathrm{E}+09$ and $2.58 \mathrm{E}+09$, respectively. At $75 \%$ duty, the mean intensities in the U87 and U373 cells were $0.595 \mathrm{E}+09$ and $1.07 \mathrm{E}+09$ compared with the control at $5.67 \mathrm{E}+09$ and $5.92 \mathrm{E}+09$, respectively. At $50 \%$ duty, mean intensities in U87 and U373 were $0.913 \mathrm{E}+08$ and $1.11 \mathrm{E}+08$, compared with the control at $1.80 \mathrm{E}+08$ and $1.95 \mathrm{E}+08$, respectively.

Characterization of the cell cycle by FACS analyses revealed that the higher the duty cycle, the higher the number 
A

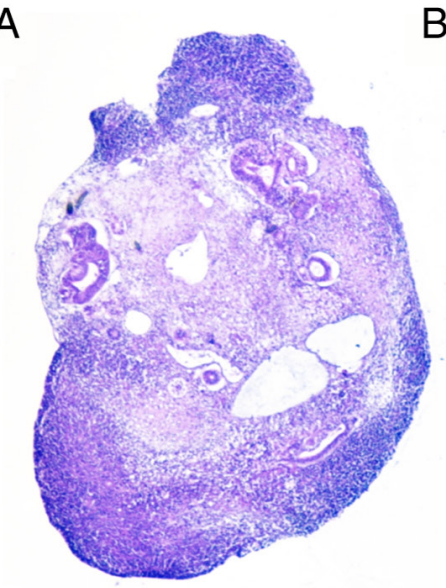

H\&E
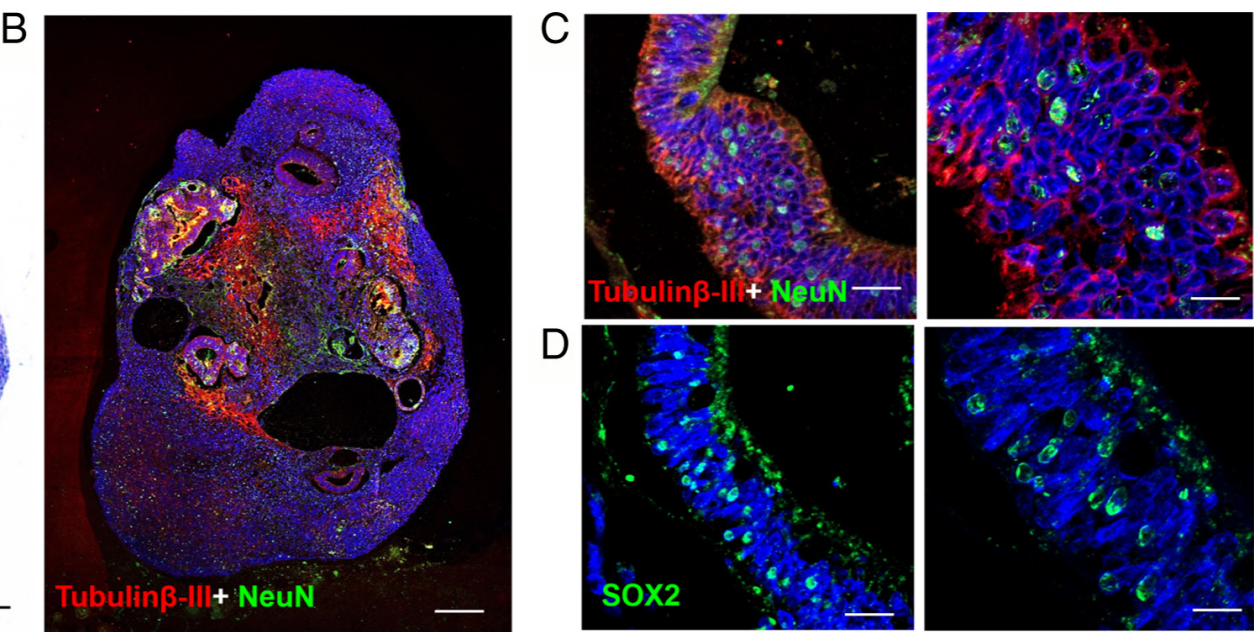

E
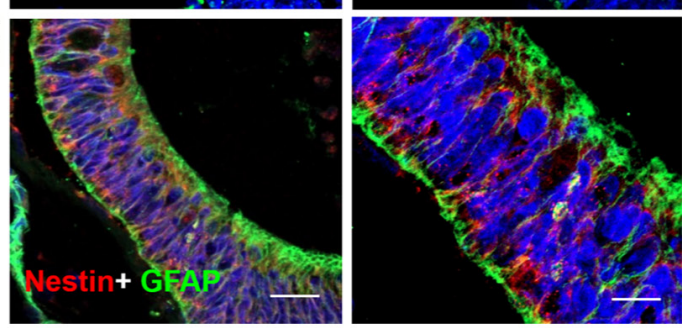

Figure 5. (A) H\&E staining of paraffin-embedded sections at 5 weeks following incubation of human brain organoid in differentiation medium. (B-E) Confocal microscopy images of hBO stained with antibodies against SOX2, Nestin, GFAP, $\beta$-III tubulin and NeuN following 5 weeks of incubation in differentiation medium. Scale bars, $50 \mu \mathrm{m}$ (A and B) and $200 \mu \mathrm{m}$ (C-E). All images are representative of two or three independent experiments.

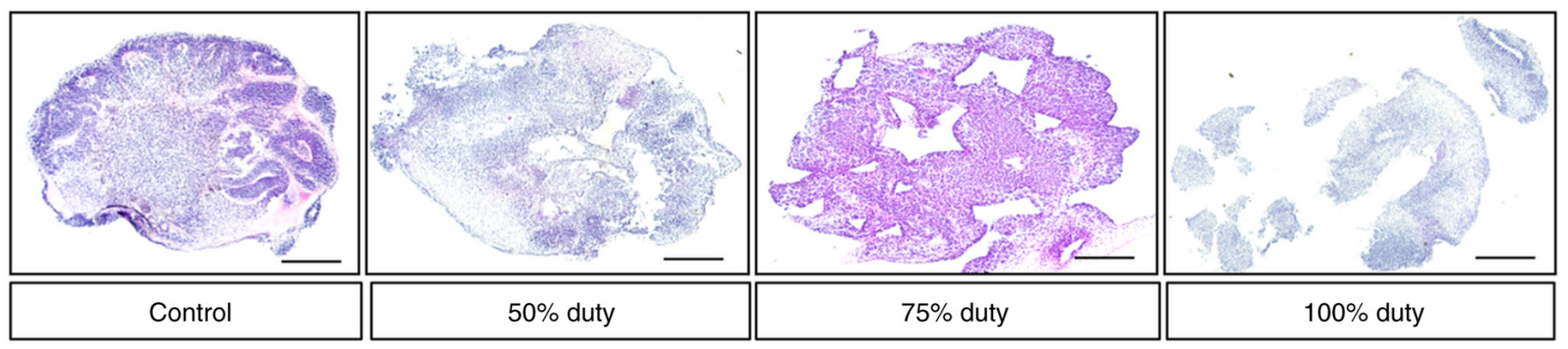

Figure 6. H\&E staining of normal brain organoids treated with tumor-treating fields for 3 days. Scale bars, $100 \mu \mathrm{m}$.

of sub-G1 cells detected (Figs. 4 and S1). Sub-G1 cells are dead cells, and they indicate apoptosis. For the U87 cells, $48.9 \%$ of the cells were in the sub-G1 phase when treated with the $100 \%$ duty TTFields application compared with 20.9 and $3.96 \%$ cells in this phase when treated with the 75 and $50 \%$ duty TTFields application, respectively. For the U251 cells, $11.9 \%$ cells were in the sub-G1 phase when treated with the $100 \%$ duty TTFields application compared with 9.54 and $1.72 \%$ cells when treated with the 75 and $50 \%$ duty TTFields application, respectively. For the U373 cells, $36.0 \%$ cells were in the sub-G1 phase when treated with the $100 \%$ duty TTFields application compared with 18.9 and $1.80 \%$ cells when treated with the 75 and $50 \%$ duty TTFields application, respectively.

TTFields application on normal brain organoids. Brain organoids were developed by culturing iPSCs under conditions that promote 3D neuroectoderm differentiation for 40 days. Brain organoids formed rosettes that were morphologically similar to ventricles. Neuroepithelium-like structures expressed the neural precursor marker, Nestin, the progenitor marker, SOX2, the neuronal markers, $\beta$-III tubulin and NeuN, and the astrocyte marker, GFAP (Figs. 5 and S2). In the control organoids, multiple rosettes were observed in the cortex of the organoid. In organoids treated with the TTFields at the 50 or $75 \%$ duty cycles, the cavity of rosettes expanded and loosened. The organoids treated with TTFields at $100 \%$ duty were disorganized (Figs. 6 and S3).

The present study then compared the expression of neuronal markers in brain organoids treated with 50,75 or $100 \%$ duty cycles for 3 days. NSC proliferation markers (Nestin) and neuronal markers ( $\beta$-III tubulin and NeuN), which indicate the differentiation and maturation of newly formed neurons, were used. At $50 \%$ duty, the expression of Nestin and $\beta$-III tubulin decreased compared with the control; however, NeuN expression was similar to that in the control. At $75 \%$ duty, the 

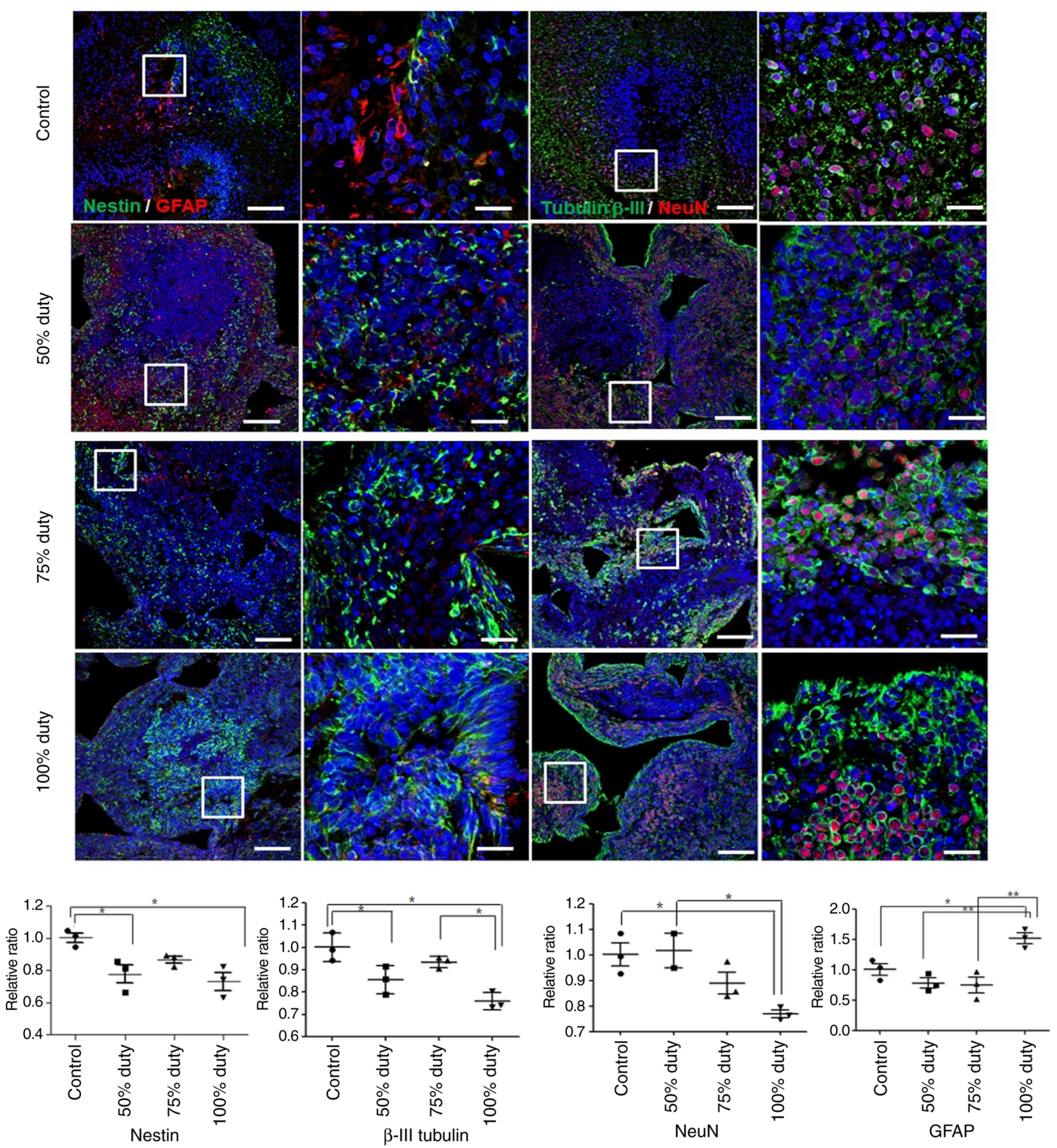

Figure 7. Confocal microscopy images of human cerebral organoids stained with antibodies against nestin, $\beta$-III tubulin, NeuN, and GFAP after tumor treating fields treatment for 3 days. Scale bars, $50 \mu \mathrm{m}$ (left column), $20 \mu \mathrm{m}$ (other columns).

expression of Nestin and $\beta$-III tubulin was similar to that in the control. However, the expression of Nestin, $\beta$-III tubulin and NeuN was significantly decreased in the brain organoids treated with TTFields at $100 \%$ duty compared with the control. By contrast, the expression of GFAP was significantly increased in the brain organoids treated with the TTFields at $100 \%$ duty. The expression of GFAP was similar in the organoids treated at the 50 and $75 \%$ duty compared with the control (Fig. 7 and Table SI).

\section{Discussion}

TTFields represent an emerging treatment modality for various tumors, including high-grade gliomas. This technology has received considerable interest as it functions based on mechanisms different from conventional cancer treatments, and thus, can be used solely or in conjunction with other therapeutic procedures. TTFields is a portable, home-usable device that can be managed via telemedicine; 
thus, it can be safely used in patients with GBM, even during the COVID-19 pandemic (30).

Although clinical results have demonstrated that TTFields inhibit tumor growth and improve overall survival (4-7), the underlying mechanisms are not fully understood. Studies have conducted in vitro experiments to reveal the mechanisms of action of TTFields; however, conventional 2D-cultured cells may not represent the essential cellular environment observed in vivo. One strategy with whch to reduce the gap between in vitro and live tissue is to use well-suited 3D-cultured cells. Spheroids are organized structures composed of several layers. The external layers are accessible to nutrients and oxygen and contain proliferative cells; the intermediate layers are composed of senescent cells; the core of the spheroid is mainly necrotic (31). The present study evaluated the effects of TTFields using three tumor spheroid cells (U87, U251 and U373) as individual GBs may respond differently to electric field exposure.

Furthermore, brain organoids were used to examine the safety of TTFields in normal cells. The safety profile of TTFields in healthy animals has also been investigated. As previously demonstrated, following a 1-month follow-up period, all animals were euthanized, and samples of the major organs were examined by a pathologist; no treatment-related toxicities were recorded in any animal (10). According to the global post-marketing safety surveillance described above (32), 52 pediatric patients (aged $<18$ years) were treated with TTFields therapy. The incidence of all adverse events was lowest in pediatric patients, most likely due to the smaller sample size. Although safety is the most important issue in pediatric clinical trials, the effects of TTF on the normal brain in pediatric patients have rarely been investigated. Cerebral organoids can serve as an informative tool for studying human neural development (33). A number of advantages of non-organoid-based in vitro models of cortical development are also applicable to brain organoids, including the ability to generate large numbers of cells, perform genetic manipulations and perform assays at multiple time points. However, knowledge of the effects of TTFields on normal brain organoids is limited. TTFields has been shown to interfere with mitosis in rapidly dividing cancer cells, thereby impairing cancer proliferation $(7,9)$. It has been reported that TTFields result in two forms of cell stress, namely, shear stress and extensile stress, that affect the cell membrane. Cancer cells are more deformable compared to non-cancer cells due to their altered membrane composition. Therefore, cancer cells' responsiveness to stress is different from that of non-cancer cells (34). To the best of our knowledge, the present study was first to address the effect of TTF on neural cells of brain organoids. The findings presented herein suggest that TTFields with the conventional parameters can lead to the apoptosis of normal brain organoids and thus merit attention for further development and clinical application. Recently, the authors used oligomeric $A \beta_{1-42}$ to induce neuronal cell death in human brain organoid cultures and found that there was greater apoptotic cell death in brain organoids cultured with oligomeric $A \beta_{1-42}$ compared to brain organoids cultured in the absence of $A \beta_{1-42}$ (unpublished data). The present study examined whether neurotoxicity was observed in brain organoids treated with TTFs at different duty cycles.
For conducting in vitro experiments, a TTFields system, including a custom-built sine wave generator was developed, which maintains constant voltage regardless of the change in cell culture medium volume and allows easy adjustment of the duty cycle. The entire system was built using readily available components with easy-to-fabricate electrodes. For system verification, computational simulations were performed, which confirmed that the experimental setup generated a uniform electric field distribution between the electrodes. Calibration testing confirmed that the system did not induce significant heating when the highest duty cycle $(100 \%)$ was applied; the temperature increase in the cell culture medium was $<0.5^{\circ} \mathrm{C}$ and was maintained at $<41^{\circ} \mathrm{C}$, which is below the temperature for thermal injury (22). The treatment effects at 100, 75 and 50\% duty were compared with those of the control. To the best of our knowledge, this is the first study to control the duty cycle of TTFields. Although clinical studies have demonstrated that patients wearing the device $>18 \mathrm{~h}$ /day experienced therapeutic effects, this is not equivalent to a prescribed duty cycle, but simply a measure of the therapeutic effect based on the wearing period; it is not a controlled study for defined duty cycles $(20,21)$. Reducing the duty cycle has advantages, such as increasing energy efficiency, thereby reducing the size and weight of the battery, and these advantages increase the possibility of transforming the device type to an insertion type or one with wireless recharging.

The results of the present study confirmed that high duty cycles of TTFields effectively induced the death of cancer cells. The evaluation of bioluminescence intensity (Fig. 3 and Table SI) revealed that the TTFields application at $75 \%$ duty inhibited cell proliferation (average $85 \%$ decrease compared to the control), which was very similar to that achieved using $100 \%$ stimulation (average $78.5 \%$ decrease compared to control), although slightly more effective. By contrast, when applying 50\% TTFields, the average reduction rate was only $43 \%$ compared to the control. The difference in the treatment effect depending on the duty cycle was more pronounced in the evaluation of the cell cycle (Fig. 4). When comparing the apoptosis phase of the TTFields group and the control group, it was found that apoptosis conspicuously increased in the 75 and $100 \%$ groups, although no significant change was observed in the $50 \%$ group. There was also a difference depending on the type of glioma spheroids, which we speculate is because of the gradients of proliferation, oxygen, nutrients, and $\mathrm{pH}$ observed from the external layer to the inner part of the spheroid $(35,36)$. However, the possible effect of the stem-cell like population in GB was not considered herein. Recent cancer treatment research has aimed to eradicate cancer stem cells as well as cancer cells, and thus further studies are required to investigate the effects of TTFields on cancer stem cells.

Of note, it was found that the expression of NSC markers decreased and that of GFAP, an astrocyte marker (37), increased following TTFields application at $100 \%$ duty. For a negative control, it was confirmed that GFAP was not expressed in kidney organoids in which GFAP-positive cells are not present (Fig. S4). In the central nervous system, astrocytes are involved in the regulation of neurodevelopment, neurotransmission, cerebral metabolism and blood 
flow $(38,39)$. In neuronal injury, astrocytes protect neurons against oxidative stress and toxicity (40). It was hypothesized that TTFields at $100 \%$ duty would affect the neuronal differentiation of normal brain organoids, and astrocyte differentiation is accelerated to escape TTF-induced stimuli. As an additional method for apoptosis detection, terminal deoxynucleotidyl transferase-mediated dUTP nick-end labeling (TUNEL) assay was performed for the analyses of glioma spheroid cells and brain organoids, as described in Data S1. It was confirmed that the apoptosis was activated at the 100 and $75 \%$ duty cycle of the TTFields in glioma spheroid cells (Fig. S1). The portion of TUNEL-positive cells in brain organoids treated with $50 \%$ and $75 \%$ duty cycle of TTFields was higher than that without TTFields exposure and brain organoids treated with $100 \%$ duty cycle of TTFields were severely disorganized (Fig. S3). There are certain limitations to the present study, involving the lack of specific markers of apoptosis. Collectively, the expression of Nestin, $\beta$-III tubulin and $\mathrm{NeuN}$ was decreased, and apoptosis occurred in the brain organoids treated with TTFields. With these findings, it was concluded that the proliferative neural cell phenotype was affected by the TTFields via the apoptotic pathway. The long-term use of $100 \%$ duty may be associated with neurotoxicity in these subjects. Duty cycle adjustment is necessary in terms of safety, in addition to energy saving. Taken together, research on neurotoxicity in the context of long-term TTFields application is required as in in the present study, TTFields exposure lasted for only 3 days.

Considering these results, it was concluded that the TTFields application at $75 \%$ duty induced cancer cell death at a similar level as the $100 \%$ duty and had less neurotoxicity. It is considered that this is an important finding for the advancement of TTFields therapy. TTFields devices have limitations in that they cause inconvenience to patients due to the size and weight of the battery, and the presence of exposed cables outside the clothes. One approach to solving this issue is to evolve the device into an implantable form using wireless recharging technology. However, to implement this, energy saving, which can be achieved through duty cycle reduction, is a key requirement for device miniaturization and operation time extension. Finally, it is considered that the present study paves the way for enhancing the usability and safety of TTFields therapy through parametric optimization. However, although the in vivo environment was closely simulated using 3D-cultured cells, there is a limitation in that it cannot perfectly reproduce the complex brain tissues composed of both cancer cells and normal brain cells. To compare the cycle of GBM spheroids and brain organoids, brain organoids and U87 cells were co-cultured under the same culture conditions (Fig. S5). From the additional experiments with co-cultured cells, it was found that the TTFields gave rise to the apoptosis of both GBM spheroids and brain organoids (Figs. 4, 6, S1 and S3). The higher the duty cycle of TTFields, the more prominent was the cell apoptosis. However, as illustrated in Figs. 6 and S3, normal brain organoids treated with TTFields were also damaged and shrunken; thus, the quantitative comparison of the cell cycle is difficult with the shape of the co-cultured cell clusters completely changing after the TTFields application. Apoptosis was observed in cell clusters not treated with TTFields, as well as with in those treated with TTFields. For the cause of this observation, it was hypothesized that cancer cells hindered the survival of brain organoids cells regardless of the effects of TTFields. In addition, it may be the result of an unsuitable cell culture medium. Since the cell culture medium is a crucial factor for cellular growth, optimizing suitable conditions for co-cultured cell clusters requires many parameter studies. Therefore, due to the confounding effects of TTFields and co-culturing, this as a limitation of the present in vitro study. The authors aim to design a TTFields system for animal experiments to investigate the effect of TTFields on cancer cells and the behavior of the animal models in a future study.

In conclusion, the present study constructed a simple TTFields system and cultured three types of cancer spheroid cells and brain organoids. Using the cancer spheroid cells, it was confirmed that the TTFields exhibited a significant anticancer effects even when duty cycle was decreased to $75 \%$. By contrast, morphological abnormalities appeared in brain organoids exposed to $100 \%$ TTFields. These findings suggest that further in-depth studies are warranted to optimize the duty cycle to enhance the safety of this emerging therapeutic modality. It is considered that the current portable, wearable form factor is suboptimal for the effectiveness and usability of TTFields therapy. Duty cycle optimization may be the first evolutionary step for TTFields systems, and the current results may serve as scientific evidence for this improvement.

\section{Acknowledgements}

Not applicable.

\section{Funding}

The present study was supported by the National Research Foundation of Korea (NRF) grant funded by the Korea government (no. 2021R1C1C201046911) and funded by the Ministry of Education (grant no. 2020R1A6A1 A03047902), and in part by the Po-Ca Networking Groups, funded by the Postech-Catholic Biomedical Engineering Institute (PCBMI) under grant no. 5-2021-B0001-00301.

\section{Availability of data and materials}

The datasets used and/or analyzed during the current study are available from the corresponding author on reasonable request

\section{Authors' contributions}

All authors contributed intellectually to the research. EY, SHY and SMP designed the experiments. EY and JEL performed the experiments and analyzed the data. The custom-built circuit was designed by YSL. The manuscript was prepared by EY and JEL. SHY and SMP reviewed the manuscript critically for important intellectual content. SHY and JEL confirm the authenticity of all the raw data. All authors have read and approved the manuscript.

\section{Ethics approval and consent to participate}

Not applicable. 


\section{Patient consent for publication}

Not applicable.

\section{Competing interests}

The authors declare that they have no competing interests.

\section{References}

1. Kirson ED, Gurvich Z, Schneiderman R, Dekel E, Itzhaki A, Wasserman Y, Schatzberger R and Palti Y: Disruption of cancer cell replication by alternating electric fields. Cancer Res 64 3288-3295, 2004.

2. Neuhaus E, Zirjacks L, Ganser K, Klumpp L, Schüler U, Zips D, Eckert $\mathrm{F}$ and Huber SM: Alternating electric fields (TTFields) activate $\mathrm{Ca}_{\mathrm{v}} 1.2$ channels in human glioblastoma cells. Cancers 11: 110,2019

3. Trusheim J, Dunbar E, Battiste J, Iwamoto F, Mohile N, Damek D, Bota DA and Connelly J: A state-of-the-art review and guidelines for tumor treating fields treatment planning and patient follow-up in glioblastoma. CNS Oncol 6: 29-43, 2017.

4. Stupp R, Wong ET, Kanner AA, Steinberg D, Engelhard H, Heidecke V, Kirson ED, Taillibert S, Liebermann F, Dbalý V, et al: NovoTTF-100A versus physician's choice chemotherapy in recurrent glioblastoma: A randomised phase III trial of a novel treatment modality. Eur J Cancer 48: 2192-2202, 2012.

5. Stupp R, Taillibert S, Kanner A, Read W, Steinberg D, Lhermitte B, Toms S, Idbaih A, Ahluwalia MS, Fink K, et al Effect of tumor-treating fields plus maintenance temozolomide vs maintenance temozolomide alone on survival in patients with glioblastoma: A randomized clinical trial. JAMA 318 : 2306-2316, 2017.

6. Stupp R, Taillibert S, Kanner AA, Kesari S, Steinberg DM, Toms SA, Taylor LP, Lieberman F, Silvani A, Fink KL, et al: Maintenance therapy with tumor-treating fields plus temozolomide vs temozolomide alone for glioblastoma: A randomized clinical trial. JAMA 314: 2535-2543, 2015.

7. Giladi M, Schneiderman RS, Voloshin T, Porat Y, Munster M, Blat R, Sherbo S, Bomzon Z, Urman N, Itzhaki A, et al: Mitotic spindle disruption by alternating electric fields leads to improper chromosome segregation and mitotic catastrophe in cancer cells. Sci Rep 5: 18046, 2015.

8. Hottinger AF, Pacheco P and Stupp R: Tumor treating fields: A novel treatment modality and its use in brain tumors. Neuro Oncol 18: 1338-1349, 2016.

9. Gera N, Yang A, Holtzman TS, Lee SX, Wong ET and Swanson KD: Tumor treating fields perturb the localization of septins and cause aberrant mitotic exit. PLoS One 10: e0125269, 2015.

10. Kirson ED, Dbalý V, Tovaryš F, Vymazal J, Soustiel JF, Itzhaki A, Mordechovich D, Steinberg-Shapira S, Gurvich Z, Schneiderman $\mathrm{R}$, et al: Alternating electric fields arrest cell proliferation in animal tumor models and human brain tumors. Proc Natl Acad Sci USA 104: 10152-10157, 2007.

11. Giladi M, Voloshin T, Shteingauz A, Munster M, Blat R, Porat Y, Schneiderman RS, Cahal S, Itzhaki A, Kirson E, et al: Alternating electric fields (TTFields) induce immunogenic cell death resulting in enhanced antitumor efficacy when combined with anti-PD-1 therapy. J Immunol 196 (Suppl 1): 75.26, 2016.

12. Silginer M, Weller M, Stupp R and Roth P: Biological activity of tumor treating fields in preclinical glioma models. Cell Death Dis 8: e2753, 2017.

13. Tuszynski JA, Wenger C, Friesen DE and Preto J: An overview of sub-cellular mechanisms involved in the action of TTFields. Int J Environ Res Public Health 13: 1128, 2016.

14. Wenger C, Miranda PC, Salvador R, Thielscher A, Bomzon Z, Giladi M, Mrugala MM and Korshoej AR: A review on tumor-treating fields (TTFields): Clinical implications inferred from computational modeling. IEEE Rev Biomed Eng 11: 195-207, 2018

15. Pampaloni F, Reynaud EG and Stelzer EH: The third dimension bridges the gap between cell culture and live tissue. Nat Rev Mol Cell Biol 8: 839-845, 2007.
16. Vinci M, Gowan S, Boxall F, Patterson L, Zimmermann M, Lomas C, Mendiola M, Hardisson D and Eccles SA: Advances in establishment and analysis of three-dimensional tumor spheroid-based functional assays for target validation and drug evaluation. BMC Biol 10: 29, 2012

17. Lancaster MA, Renner M, Martin CA, Wenzel D, Bicknell LS, Hurles ME, Homfray T, Penninger JM, Jackson AP and Knoblich JA: Cerebral organoids model human brain development and microcephaly. Nature 501: 373-379, 2013.

18. Di Lullo E and Kriegstein AR: The use of brain organoids to investigate neural development and disease. Nat Rev Neurosci 18: 573-584, 2017.

19. Kanner AA, Wong ET, Villano JL, Ram Z and EF-11 Investigators: Post-hoc analyses of intention-to-treat population in phase III comparison of NovoTTF-100A $\mathrm{A}^{\mathrm{TM}}$ system versus best physician's choice chemotherapy. Semin Oncol 41 (Suppl 6): S25-S34, 2014.

20. Benson L: Tumor treating fields technology: Alternating electric field therapy for the treatment of solid tumors. Semin Oncol Nurs 34: 137-150, 2018.

21. Lacouture ME, Davis ME, Elzinga G, Butowski N, Tran D, Villano JL, DiMeglio L, Davies AM and Wong ET: Characterization and management of dermatologic adverse events with the NovoTTF-100A System, a novel anti-mitotic electric field device for the treatment of recurrent glioblastoma. Semin Oncol 41 (Suppl 4): S1-S14, 2014.

22. Toms SA, Kim CY, Nicholas G and Ram Z: Increased compliance with tumor treating fields therapy is prognostic for improved survival in the treatment of glioblastoma: A subgroup analysis of the EF-14 phase III trial. J Neurooncol 141: 467-473, 2019.

23. Berkelmann L, Bader A, Meshksar S, Dierks A, Majernik GH, Krauss JK, Schwabe K, Manteuffel D and Ngezahayo A: Tumour-treating fields (TTFields): Investigations on the mechanism of action by electromagnetic exposure of cells in telophase/cytokinesis. Sci Rep 9: 7632, 2019.

24. Plonsey R and Heppner DB: Considerations of quasi-stationarity in electrophysiological systems. Bull Math Biophys 29: 657-664, 1967.

25. Pennes HH: Analysis of tissue and arterial blood temperatures in the resting human forearm. J Appl Physiol (1985) 85: 5-34, 1998.

26. Korshoej AR, Hansen FL, Thielscher A, von Oettingen GB and Sørensen JC: Impact of tumor position, conductivity distribution and tissue homogeneity on the distribution of tumor treating fields in a human brain: A computer modeling study. PLoS One 12: $\mathrm{e} 0179214,2017$.

27. Yang SH, Hong YK, Jeun SS, Kim IS, Hong JT, Sung JH, Son BC, Lee SW, Kim MC and Lee KS: Assessment of cetuximab efficacy by bioluminescence monitoring of intracranial glioblastoma xenograft in mouse. J Neurooncol 95: 23-28, 2009.

28. Kim Y, Park N, Rim YA, Nam Y, Jung H, Lee K and Ju JH: Establishment of a complex skin structure via layered co-culture of keratinocytes and fibroblasts derived from induced pluripotent stem cells. Stem Cell Res Ther 9: 217, 2018.

29. Maxim Integrated: Programmable Resolution 1-Wire Digital Thermometer. Maxim Integrated Products, Inc., San Jose, CA, 2019. https://datasheets.maximintegrated.com/en/ds/DS18B20.pdf.

30. Gatson NT, Barnholtz-Sloan J, Drappatz J, Henriksson R, Hottinger AF, Hinoul P, Kruchko C, Puduvalli VK, Tran DD, Wong ET, et al: Tumor treating fields for glioblastoma therapy during the COVID-19 pandemic. Front Oncol 11: 679702, 2021.

31. Mehta G, Hsiao AY, Ingram M, Luker GD and Takayama S: Opportunities and challenges for use of tumor spheroids as models to test drug delivery and efficacy. J Control Release 164: 192-204, 2012.

32. Shi W, Blumenthal DT, Oberheim Bush NA, Kebir S, Lukas RV, Muragaki Y, Zhu JJ and Glas M: Global post-marketing safety surveillance of tumor treating fields (TTFields) in patients with high-grade glioma in clinical practice. J Neurooncol 148: 489-500, 2020

33. Lewis EM, Kaushik K, Sandoval LA, Antony I, Dietmann S and Kroll KL: Epigenetic regulation during human cortical development: Seq-ing answers from the brain to the organoid. Neurochem Int 147: 105039, 2021.

34. Aguilar AA, Ho MC, Chang E, Carlson KW, Natarajan A Marciano T, Bomzon Z and Patel CB: Permeabilizing cell membranes with electric fields. Cancers (Basel) 13: 2283, 2021.

35. Carlsson $\mathrm{J}$ and Acker $\mathrm{H}$ : Relations between $\mathrm{pH}$, oxygen partial pressure and growth in cultured cell spheroids. Int J Cancer 42: 715-720, 1988. 
36. Khaitan D, Chandna S, Arya MB and Dwarakanath BS: Establishment and characterization of multicellular spheroids from a human glioma cell line; Implications for tumor therapy. J Transl Med 4: 12, 2006.

37. Dezonne RS, Sartore RC, Nascimento JM, Saia-Cereda VM, Romão LF, Alves-Leon SV, Souza JM, Martins-de-Souza D, Rehen SK and Gomes FC: Derivation of functional human astrocytes from cerebral organoids. Sci Rep 7: 45091, 2017.

38. Siracusa R, Fusco R and Cuzzocrea S: Astrocytes: Role and functions in brain pathologies. Front Pharmacol 10: 1114, 2019.
39. Khakh BS and Sofroniew MV: Diversity of astrocyte functions and phenotypes in neural circuits. Nat Neurosci 18: 942-952, 2015.

40. Ouyang YB, Xu L, Lu Y, Sun X, Yue S, Xiong XX and Giffard RG: Astrocyte-enriched miR-29a targets PUMA and reduces neuronal vulnerability to forebrain ischemia. Glia 61: 1784-1794, 2013.

c) (i) $\ominus$ This work is licensed under a Creative Commons Attribution-NonCommercial-NoDerivatives 4.0 International (CC BY-NC-ND 4.0) License. 